\title{
Calcul de la propagation de la marée dans une zone côtière
}

\section{Calculation of tidal propagation in a coastal zone}

PAR

\author{
R. BONNEFILLE ET F. VOYER \\ INGÉNIEURS A L'ẺLECTRICITÉ DE FRANGE
}

\begin{abstract}
Détermination du remous de l'usine marémotrice des îles Chansey pour différents types de fonctionnement.

Etude, soit ḋ l'aide de machines à calculer, soil à l'aide du modèle réduit, de l'influence sur la marée d'un débit variant sinusoüdalement en fonction du temps et injecté en divers points de l'usine, et ceci dans diverses hypotheses concernant la prise en compte de la force de Coriolis ou de la pente du fond de la mer. Résultats principaux et confrontation des données du modèle réduit et du calcul.
\end{abstract}

\begin{abstract}
Determination of the backwater curve of the Chausey Islands tidal plant for various types of operation.

Use of calculating machines or a scale model to study how the tide is affected by a flow injected into the plant at various points, at a rate varying sinusoidally with time, for various assumptions allowing for Coriolis force and the slope of the sea bed.

Principal results, and a comparison between the model data and calculated results.
\end{abstract}

\section{I. - INTRODUCTION}

L'usine marémotrice de la Rance, dont la construction vient d'être entreprise par l'Electricité de France, comportera un bassin dont la surface moyenne est de $15 \mathrm{~km}^{2}$. Un projet beaucoup plus ambitieux envisage l'équipement dans le golfe de Saint-Malo d'un bassin atteignant $600 \mathrm{~km}^{2}$, c'est-à-dire quarante fois plus important que celui de la Rance. Les ouvrages, longs d'une vingtaine de kilomètres, s'étendront de la Pointe du Grouin près de Cancale à la presqu'île du Cotentin en s'appuyant sur l'archipel des îles Chausey (fig. 1). Ils comporteront, d'une part, les turbines et les vannes assurant les échanges de débit entre le bassin et la mer, d'autre part, les digues étanches de fermeture du bassin. La puissance installée sera de 8 à 12 millions de $\mathrm{kW}$.

L'ampleur du projet des îles Chausey pose des problèmes complexes de construction et d'exploitation, et de plus, l'influence de l'usine sur le régime de la marée peut ne plus être négligeable et doit être étudiée avec soin. Cette étude, entreprise il y a quelques années, se poursuit à la fois par des méthodes de calculs numériques et à l'aide d'essais sur modèle réduit. La confrontation des résultats est extrêmement féconde. Dans le cas particulier, le calcul permet de prendre facilement en compte l'accélération complémentaire due à la rotation de la terre, qui n'est pas représentée à l'échelle dans la technique classique de construction des modèles réduits fixes.

Le problème théorique $d u$ remous d'un ouvrage dans une mer à marée a été étudié de façon très complète par $M$. Vantroys dans sa thèse de doctorat publiée dans les Annales Hydrographiques de 1958. Le problème étudić cst le suivant : connaissant d'une part le régime actuel de la marée dans une région déterminée, et d'autre part, dans le cas d'une usine marémo- 


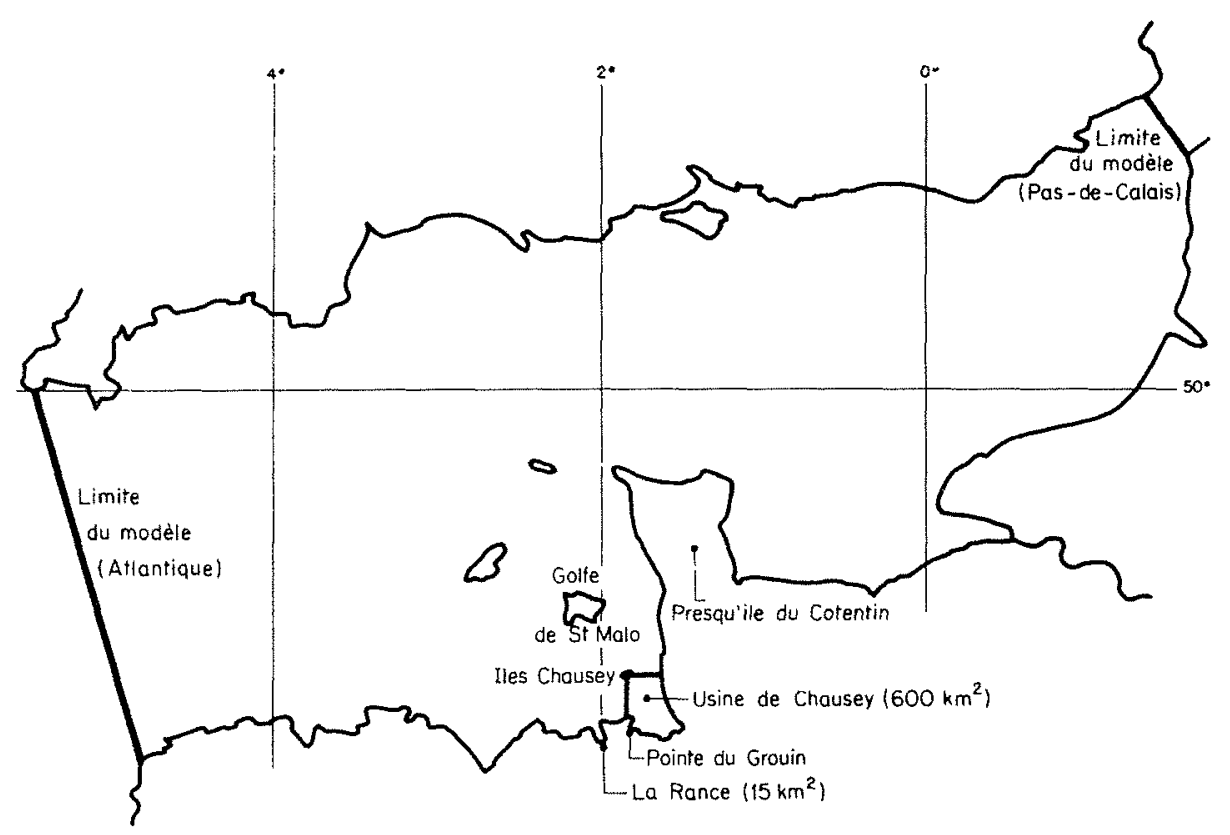

Fra. 1

Carte de la Manche.

trice, connaissant le tracé de la digue et le programme d'exploitation défini par le débit de l'usine en fonction du temps, quelle est la perturbation du champ des dénivellations et des courants de la marée créée par l'exploitation de l'usine?

Une hypothèse importante et approximativement vérifiée est que le problème est représentable par des relations Jinéaires. Le champ de perturbations peut alors être étudié indépendamment de la maréc, à Iaquelle il se superpose. Nous supposerons donc que la mer est immobile au niveau moyen. De même, il sera possible d'étudier la perturbation apportée par des éléments d'usine fonctionnant séparément, la superposition des solutions élémentaires permettant d'obtenir la perturbation totale apportée par l'usine complète. Dans ce but, nous avons séparé en trois parties chacune des digues GrouinChausey et Chausey-Cotentin (fig. 1), chacun des éléments d'usine étant exploité séparément suivant une loi de débit sinusoïdal d'amplitude donnée. Rappelons en effet que l'exploitation d'une usine marémotrice aboutit essentiellement à modifier la phase existant dans la marée naturelle entre l'amplitude de la marée (le potentiel) et le courant de la marée (l'intensité). Le modèle réduit permet de vérifier et éventuellement d'adapter la schématisation admise pour les besoins du calcul et c'est ainsi que les premiers essais sur modèle ont confirmé la linéarité des phénomènes pour des débits de $400000 \mathrm{~m}^{3} / \mathrm{s}$, c'est-à-dire de l'ordre de grandeur de ceux envisagés pour l'usine marémotrice des îles Chausey.

En conclusion, le but de nos études est d'obtenir des ordres de grandeurs des phénomènes étudiés et de connaître l'influence, sur ces phénomènes, des nombreux paramètres à prendre en compte: accélération complémentaire de Coriolis, rugosité, conditions aux limites, etc.

\section{II. - LES EQUATIONS DU PROBLEME}

Les calculs intéressent une surface déterminée de la mer choisie suffisamment grande pour que la perturbation du côté du large puisse être considérée comme négligeable. Cette hypothèse pourra être vérifiée a posteriori.

Les notations sont les suivantes:

$$
\begin{array}{r}
h(x, y) \text { représente la profondeur de la } \\
\text { mer rapportée au niveau moyen; }
\end{array}
$$

$\zeta$ est l'amplitude de la dénivellation comptée par rapport au niveau moyen;

$\vec{V}$ est la vitesse du courant de composantes $n$ et $v$, puisque l'étude est limitée à celle des mouvements de masse pour lesquels les vitesses verticales et les gra- 
dients verticaux des vitesses horizontales peuvent être négligés;

$\gamma=2 \omega \sin \mathrm{L}$ est un facteur introduit par l'accélération de Coriolis, w étant la vitesse de rotation de la terre et L la latitude;

$\vartheta \vec{V}$ est un terme qui caractérise les résistances passives selon les notations de Walter Hansen;

$h_{x}$ et $h_{y}$ sont les dérivées partielles de $h$ par rapport à $x$ et $y$, permettant de prendre en compte les variations de la pente du fond.

Compte tenu de ces remarques, l'équation dynamique se traduit par :

$$
\begin{aligned}
& g \zeta_{x}+u_{t}+p u-\gamma v=0 \\
& g \zeta_{y}+v_{t}+\rho v+\gamma u=0
\end{aligned}
$$

et l'équation de continuité par :

$$
h\left(u_{x}+v_{y}\right)+h_{x} u+h_{y} v+\zeta_{t}=0
$$

Si l'on se borne à l'étude de perturbation sinusoïdale de pulsation $\alpha$, on obtient, par élimination de $u$ et $v$ entre les équations (1), (2) et (3) et en adoptant la représentation par des exponentielles imaginaires, l'équation (E) aux déri- vées partielles du second ordre, qu'il s'agit d'intégrer dans un certain domaine.

$$
\begin{aligned}
g h\left(\zeta_{x^{2}}+\zeta_{y^{2}}\right) & +g\left(h_{x}-\frac{\gamma}{\rho+j \alpha} h_{y}\right) \zeta_{x} \\
& +g\left(h_{y}+\frac{\gamma}{\rho+j \alpha} h_{x}\right) \zeta_{y} \\
& -\frac{j \alpha}{1+j \alpha}\left[(\rho+j \alpha)^{2}+\gamma^{2}\right] \zeta=0
\end{aligned}
$$

avec $j=\sqrt{-1}$.

A la limite du contour fermé entourant complètement la zone de définition de l'inconnue $\zeta$, les conditions sont de trois sortes:

a) Du côté du large, on admet que la marée n'est pas perturbée, donc que la perturbation étudiée est nulle ou négligeable.

$$
\zeta=0
$$

Cette condition devra se justifier a posteriori par les conclusions auxquelles conduira le calcul.

b) Le long de la côte, la composante normale du courant est nulle;

c) Le long des ouvrages, la loi de débit est supposée connue.

\section{III. - LES CALCULS NUMERIQUES}

Dans la recherche d'une solution numérique, les équations aux dérivées partielles sont remplacées par des équations aux différences finies définies en un nombre de points d'un canevas orienté suivant les axes $x$ et $y$. Si l'on désigne par $N$ le nombre total des points du canevas (points intérieurs et points frontières), nous obtenons $\mathrm{N}$ équations linéaires entre les $\mathrm{N}$ inconnues qui sont les valeurs de $\zeta$ en ces $N$ points. II s'agit d'un système de $\mathrm{N}$ équations à $\mathrm{N}$ inconnues complexes qui peut être explicité sous forme de $2 \mathrm{~N}$ équations à $2 \mathrm{~N}$ inconnues réelles.

La méthode utilisée pour résoudre pratiquement ce problème a évolué dans le temps en même temps que les possibilités des différents calculateurs utilisés. Nous allons décrire rapidement les différentes étapes des calculs, pour arriver à la. dernière méthode mettant en œuvre des moyens puissants.

1. Une première série de calculs a été effectuée en 1956 par le Service mathématique des Etudes et Recherches sur la calculatrice dont dis- posait alors ce Service. Le maillage adopté était uniforme et de $5 \mathrm{~km}$ de côté, il s'étend du Cotentin à l'est jusqu'au méridien du golfe de SaintBrieuc à l'ouest. Il était limité au nord par le parallèle de Guernesey. Le calcul effectué prenait en compte ou non l'accélération de Coriolis, mais la variation de la pente était négligée.

Un calcul effectué par itérations sur le canevas comportant 219 points a été exposé dans une communication de M. Carteron aux IVes Journées de l'Hydraulique. Mais ce calcul itératif, imposé par les possibilités du calculateur, est très long et la convergence n'est pas assurée dans tous les cas. De plus, l'introduction de modifications de méthode assurant la convergence, allongeraient les calculs au point de rendre la méthode pratiquement inutilisable.

2. En 1957, la puissance accrue des machines à la disposition du Service mathématique d'Electricité de France, ont permis, pour une durée de calculs acceptable ( 1 jour), un calcul direct par inversion de matrice jusqu'à l'ordre 57. 
Le schéma adopté à titre r'essai comportait alors pour la même surface géographique que précédemment, un quadrillage non uniforme de 56 points. Les carreaux avaient $5 \mathrm{~km}$ de côté au voisinage de l'usine et atteignaient $20 \mathrm{~km}$ à la limite extérieure côté mer. Les résultats obtenus ont montré que la représentation était trop schématique et qu'elle distordait considérablement le problème éludié, surtout dans les régions situées an nord de Jersey.

3. A cet essai, a succédé un nouveau calcul par inversion de matrice sur un canevas à 216 points presque identique au canevas à 219 points adopté pour les calculs par itérations. Ce calcul a été effectué en découpant la matrice en sousmatrices, l'ordre de ces dernières restant inférieur à 57 . Les résultats de ce calcul ont fait apparaître une anomalie dans la forme des courbes d'égale amplitude de la perturbation.

Dans ce dernier calcul, l'accélération de Coriolis n'avait pas été prise en compte, puisqu'il avait pour but l'étalonnage du canevas et de la méthode de calculs.

4. Les modalités d'exécution de ces premiers calculs étaient très lourdes, mais l'apparition de nouvelles calculatrices plus puissantes a apporté de nouvelles possibilités. Nous nous proposons ici de décrire succinctement la méthode et les résultats obtenus sur ordinateur IBM 704.

Le canevas adopté est identique au précédent : canevas uniforme à 216 points. En chaque point de ce réseau, on connaît les valeur's des coefficients complexes de l'équation (E) fonction de la configuration du point considéré (profondeur, pente du fond, frottement, courant, etc.), et de l'accélération de Coriolis, et l'on se propose de déterminer la fonction complexe $\zeta(x, y)$ résultant d'une perturbation. Cette perturbation est l'injection d'un débit sinusoïdal correspondant à une vitesse de $1 \mathrm{~m} / \mathrm{s}$ successivement en chacun des six points schématisant les éléments de l'usine. La vitesse de $1 \mathrm{~m} / \mathrm{s}$ représente pour chaque élément d'usine un débit de l'ordre de
$125000 \mathrm{~m}^{3} / \mathrm{s}$ pour les points 2 et 3 , ct de $50000 \mathrm{~m}^{3} / \mathrm{s}$ pour les points $1,4,5$, et 6 .

La matrice du système proposé est remarquable par le fait qu'elle est très creuse; en effet, chaque point n'est relié en fait qu'à un petit nombre (quatre ou cing) de points voisins. Il serait possible de traiter globalement cette première matrice, mais la durée de calculs serait de 4 à $5 \mathrm{~h}$. Or une durée de calcul de 1 h 30 a pu être obtenue en calculant une deuxième matrice dite «matrice triangularisée », compte tenu de la particularité de la matrice initiale d'être très creuse.

Pratiquement le calcul comporte les points suivants :

\section{Premier PASSAGE EN MAGHiNe :}

$1^{\circ}$ Calcul des coefficients de la matrice - sortie sur bandes;

\section{DEUXIÈme PASSAGE EN MACHINE :}

$2^{\circ}$ Transformation en matrice triangulaire;

$3^{\circ}$ Résolution du système triangulaire et sortie sur bandes pour exploitation;

\section{$4^{\circ}$ Calcul des résidus.}

La sortie sur bandes du point (3) permet de donner les résultats relatifs aux six injections de l'usine, directement en amplitude et en phase. Les résidus calculés dans tous les cas permettent d'apprécier la précision des calculs.

De plus, la durée de calcul machine de 1 h 30 mentionnée plus haut concerne, pour un canevas déterminé, d'une part les résultats relatifs aux six injections de l'usine, et également d'autre part les résultats relatifs à 31 points de la limite du large où on a successivement admis une perturbation sinusoïdale de niveau d'amplitude $\zeta=$ $1 \mathrm{~m}$. Dans les hypothèses de linéarité adoptées, la superposition de ces derniers calculs élémentaires affectés de coefficients tenant compte de la marée naturelle sur tout le contoux doit permettre de retrouver la marée naturelle dans tout le domaine considéré.

\section{IV. - LES RESULTATS DES CALCULS NUMERIQUES}

Deux séries de calculs ont été effectuées : l'une, dans le cas le plus général, tient compte de la variation de la pente $\mathrm{du}$ fond, elle comprend un calcul avec l'accélération de Coriolis et un calcul sans l'accélération de Coriolis. L'autre série de calculs ne tient pas compte de la pente du fond; elle comprend également un calcul avec l'accélération de Coriolis et un calcul sans l'accélération de Coriolis. En chaque point du canevas à 216 points ont été calculés, d'une part, la demi-amplitude de la perturbation du niveau de la mer pour un courant de $1 \mathrm{~m} / \mathrm{s}$ injecté à travers une partie donnée de l'usine et, d'autre part, le déphasage en temps entre l'heure du maximum de la perturbation et l'heure du maximum de courant injecté dans le sens bassin-mer 


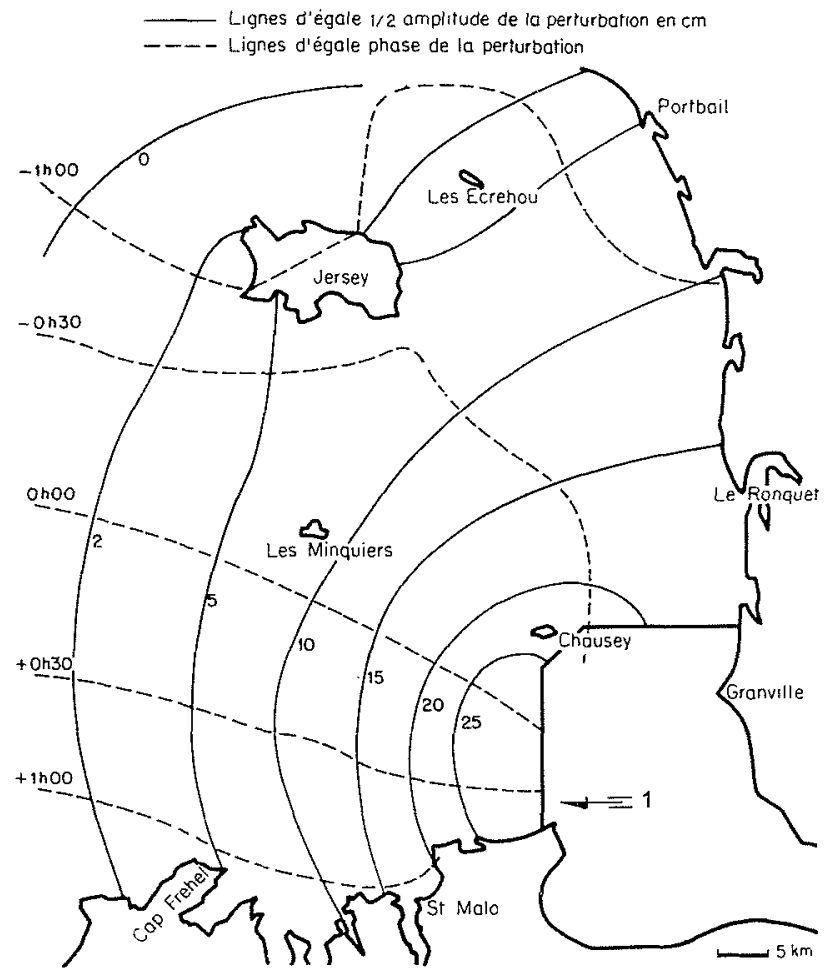

FIG. 2

Influence, sur le niveau moyen de la mer, d'un courant de $1 \mathrm{~m} / \mathrm{s}$ in jecté à travers la partie d'usine $\mathrm{n}^{\mathbf{0}} 1$. Résultats du calcul en tenant compte de la variation de la pente du fond et de la force de Coriolis.

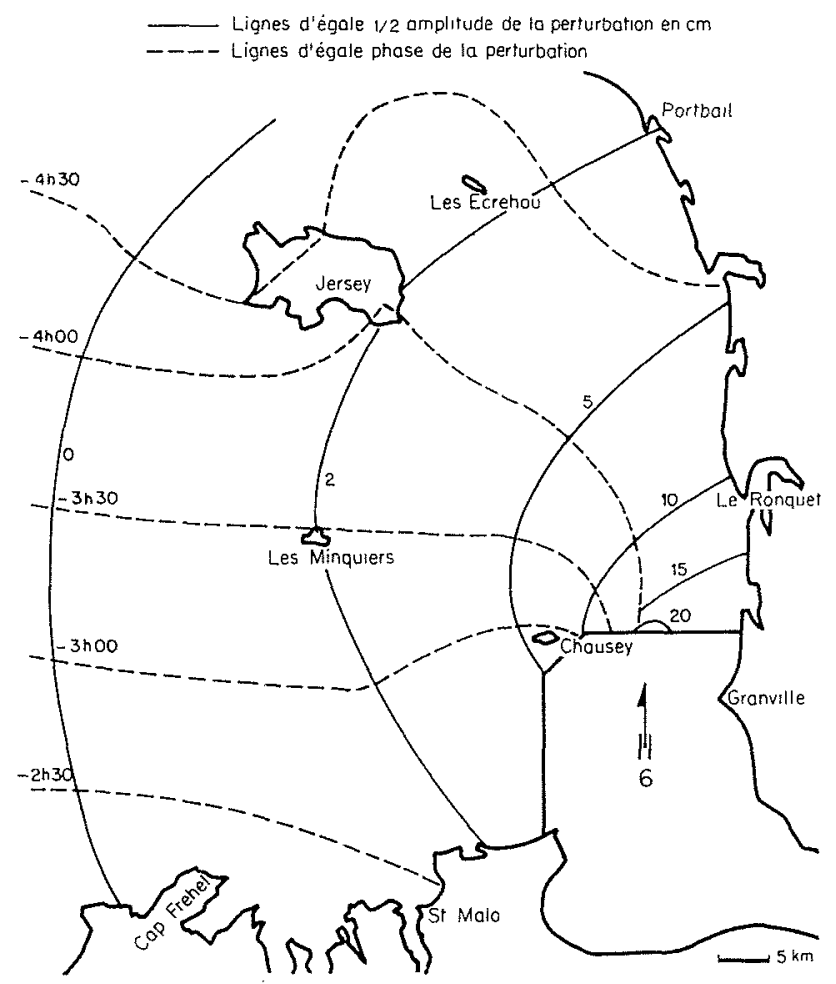

Fro. 4

Influence, sur le niveau moyen de la mer, d'un courant de $1 \mathrm{~m} / \mathrm{s}$ injecté à travers la partie d'usine $n^{\circ} 6$. Résultats du calcul en tenant compte de la variation de la pente $\mathrm{du}$ fond et de la force de Coriolis.

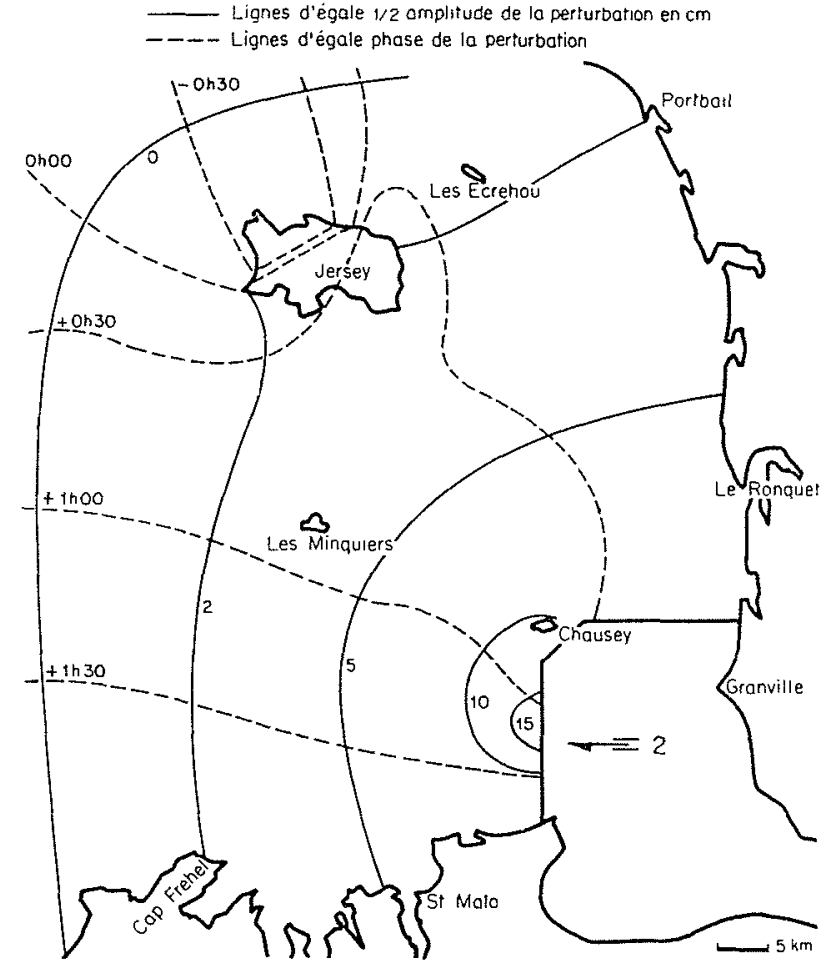

FIG. 3

Influence, sur le niveau moyen de la mer, d'un courant de $1 \mathrm{~m} / \mathrm{s}$ injecté à travers la partie d'usine $\mathrm{n}^{\circ} 2$. Résultats du calcul en tenant compte de la variation de la pente du fond et de la force de Coriolis.

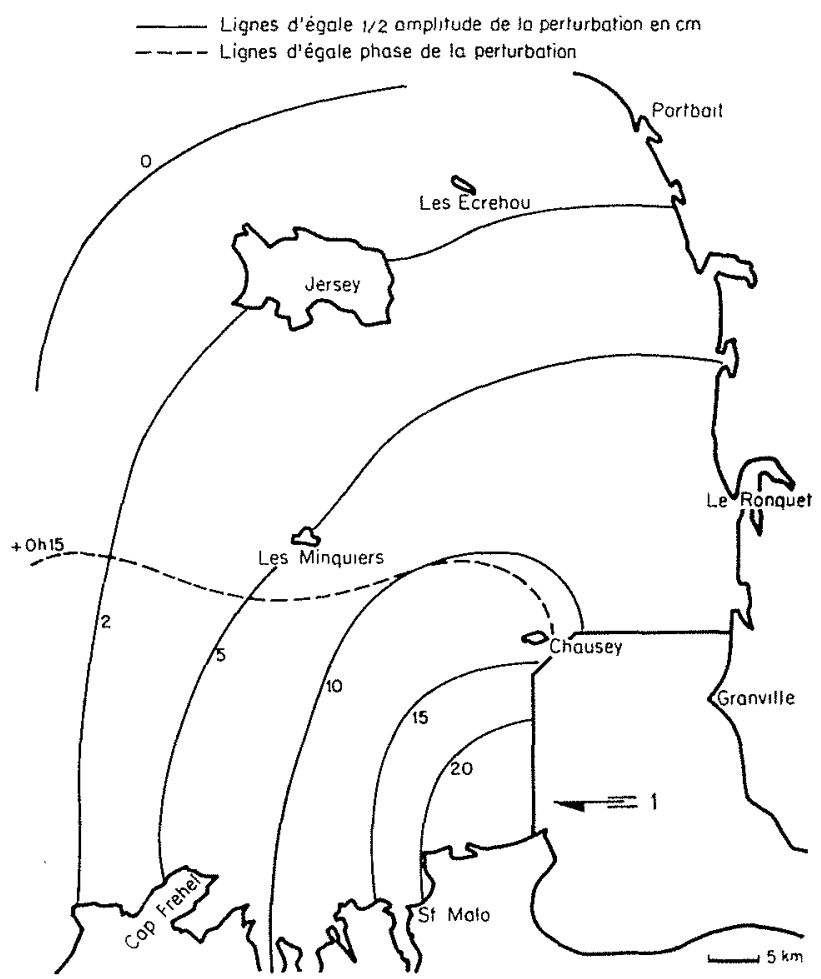

Fig. 5

Influence, sur le niveau moyen de la mer, d'un courant de $1 \mathrm{~m} / \mathrm{s}$ injecté à travers la partie d'usine $\mathrm{n}^{\circ} 1$. Résul tats du calcul en tenant compte de la variation de la pente du fond, mais en négligeant la force de Coriolis. 
à travers la partie de l'usine considérée. Cette grandeur est affectée du signe + ou - suivant que la perturbation est en avance ou en retard sur l'heure du maximum de courant. La durée de la période du mouvement est égale à $12 \mathrm{~h} 24 \mathrm{mn}$. L'heure 0 correspond à l'instant du maximum de courant dans le sens bassin-mer.

A partir de ces résultats, point par point, ont été tracées les lignes d'égale demi-amplitude en trait plein et cotées en centimètres et les lignes d'égal déphasage en trait discontinu, cotées en heures et minutes et affectées d'un signe. Nous n'avons reproduit ici que les graphiques relatifs aux injections aux points 1,2 et 6, puisqu'en effet, d'une part, les résultats relatifs aux points 3 et 2 sont très voisins et que d'autre part, il en est de même pour les résultats relatifs aux points d'injection 5 et 7 qui ont la même allure générale que ceux obtenus avec l'élément d'usine $n^{\circ} 6$ en fonctionnement.

Les figures 2,3 et 4 sont relatives aux résultats où l'on tient compte de la variation de la pente du fond et de l'accélération de Coriolis, tandis que les figures 5,6 et 7 correspondent au cas où l'on néglige la force de Coriolis. Enfin les figures 8 à 13 présentent les résultats du calcul où l'on ne tient pas compte de la variation de la pente du fond avec et sans force de Coriolis.

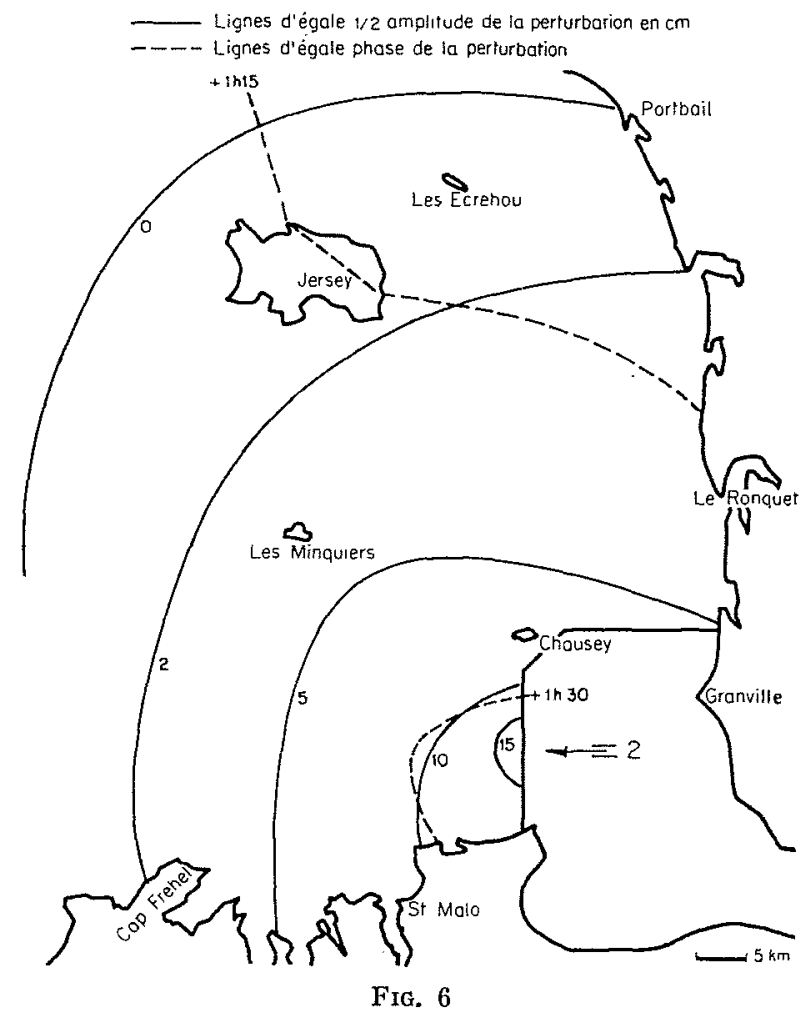

Influence, sur le niveau moyen de Ia mer, d'un courant de $1 \mathrm{~m} / \mathrm{s}$ injecté à travers la partie d'usine $n^{\circ} 2$. Résultats du calcul en tenant compte de la variation de la pente du fond, mais en négligeant la foree de Coriolis-

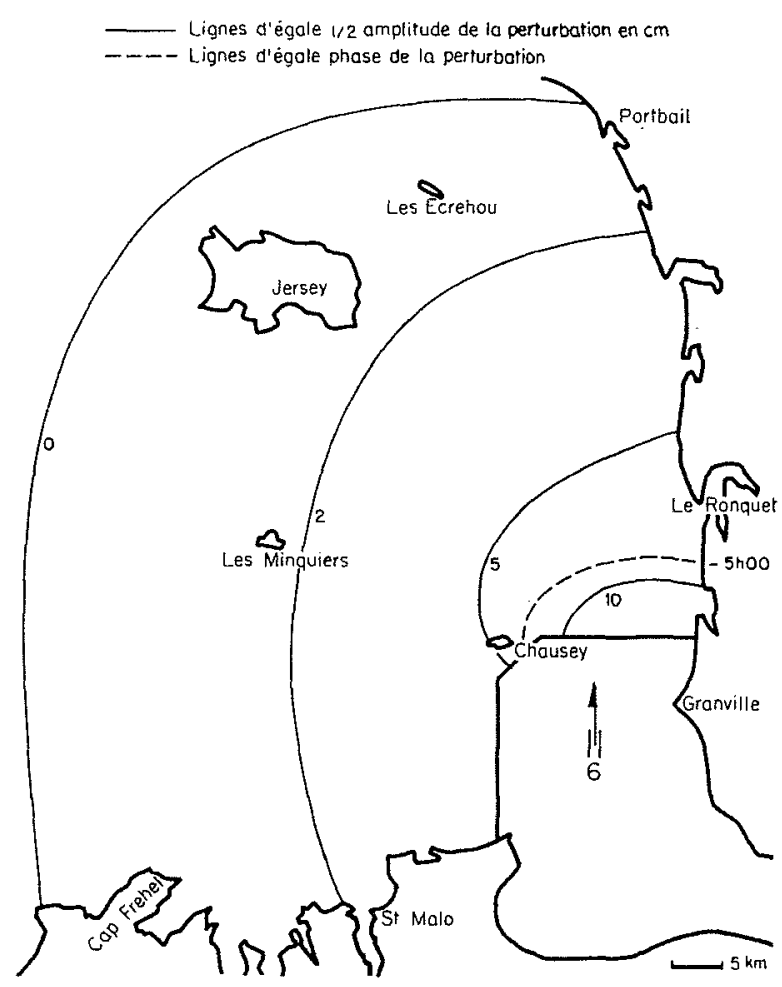

FYG. 7

Influence, sur le niveau moyen de la mer, d'un courant de $1 \mathrm{~m} / \mathrm{s}$ injecté à travers la partie d'usine $n^{\circ} 6$. Résultats du calcul en tenant compte de la variation de la pente du fond, mais en négligeant la force de Coriolis.

Les comparaisons respectives des figures 2 et 5,3 et 6,4 et 7 , etc. pour lesquelles la seule différence est la présence ou l'absence de la force de Coriolis, montrent que cette dernière a peu d'influence en ce qui concerne l'amplitude de la perturbation. En règle générale cependant, l'amplitude de la perturbation au voisinage de l'usine est plus grande lorsqu'on prend en compte l'accélération de Coriolis, sauf pour les figures 9 et 12 .

Par contre, les réseaux de lignes d'égale phase de la perturbation sont très différents suivant que l'on tient compte ou non de la force de Coriolis. En l'absence de force de Coriolis, la perturbation se déplace dans le domaine du calcul en $1 / 2 \mathrm{~h}$ environ. Cette onde semble prendre naissance au point d'injection de l'usine environ $1 \mathrm{~h}$ avant le maximum de débit pour les points $n^{\text {os }} 1$, 2 et 3 sur la face ouest de l'usine (fig. 6 et 12). Dans le cas oì l'on tient compte de la force de Coriolis, la perturbation semble se propager toujours du sud vers le nord et ceci quelle que soit la position du point d'injection du débit. Elle traverse le domaine de calcul en un temps de l'ordre de $2 \mathrm{~h}$.

On peut essayer d'expliquer ces résultats, compte tenu de nos études expérimentales sur l'influence de la force de Coriolis. D'une part, 


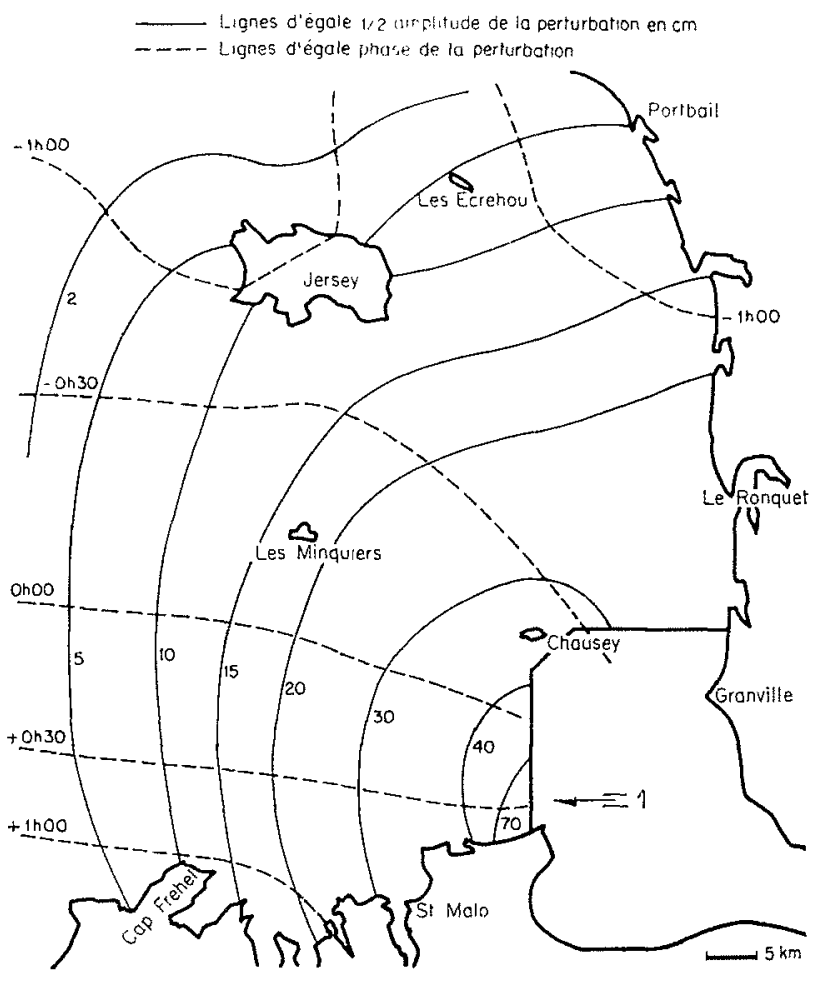

FIG. 8

Influence, sur le niveau moyen de la mer, d'un courant de $1 \mathrm{~m} / \mathrm{s}$ injecté à travers Ia partie d'usine $n^{\circ} 1$. Résultats du calcul en négligeant la variation de la pente du fond, mais en tenant compte de la force de Coriolis.

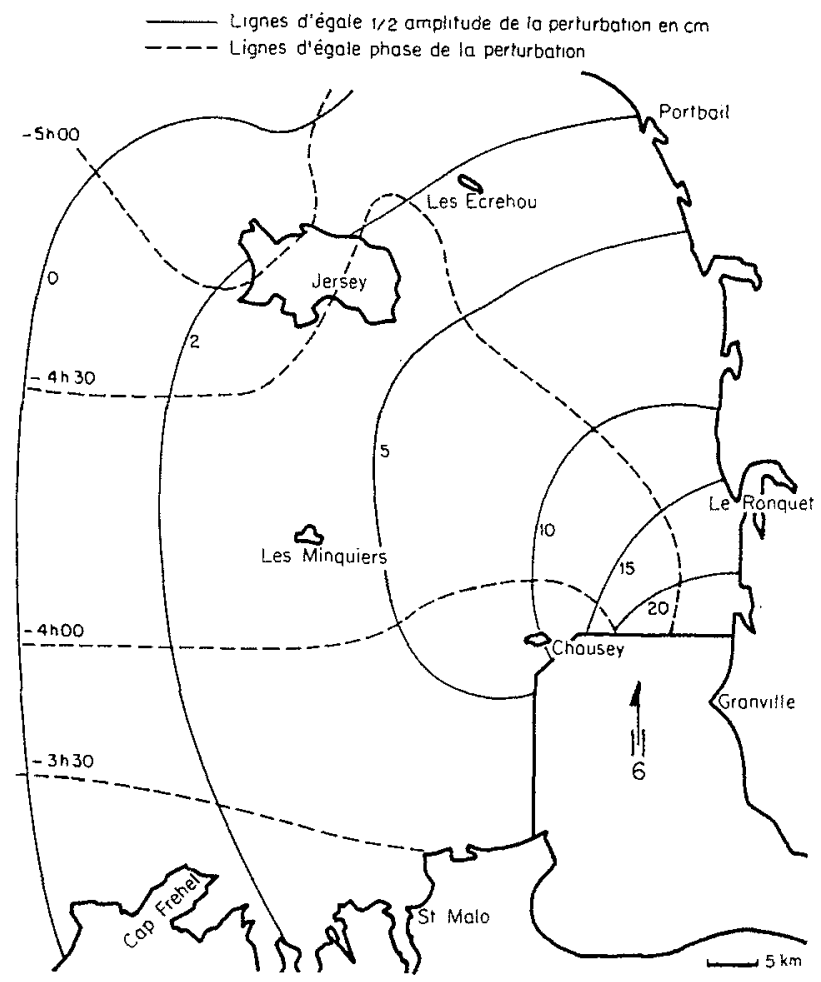

FIG. 10

Influence, sur le niveau moyen de la mer, d'un courant de $1 \mathrm{~m} / \mathrm{s}$ injecté à travers la partie d'usine $\mathrm{n}^{\circ} 6$. Résultats du calcul en négligeant la variation de la pente du fond, mais en tenant compte de la force de Coriolis.

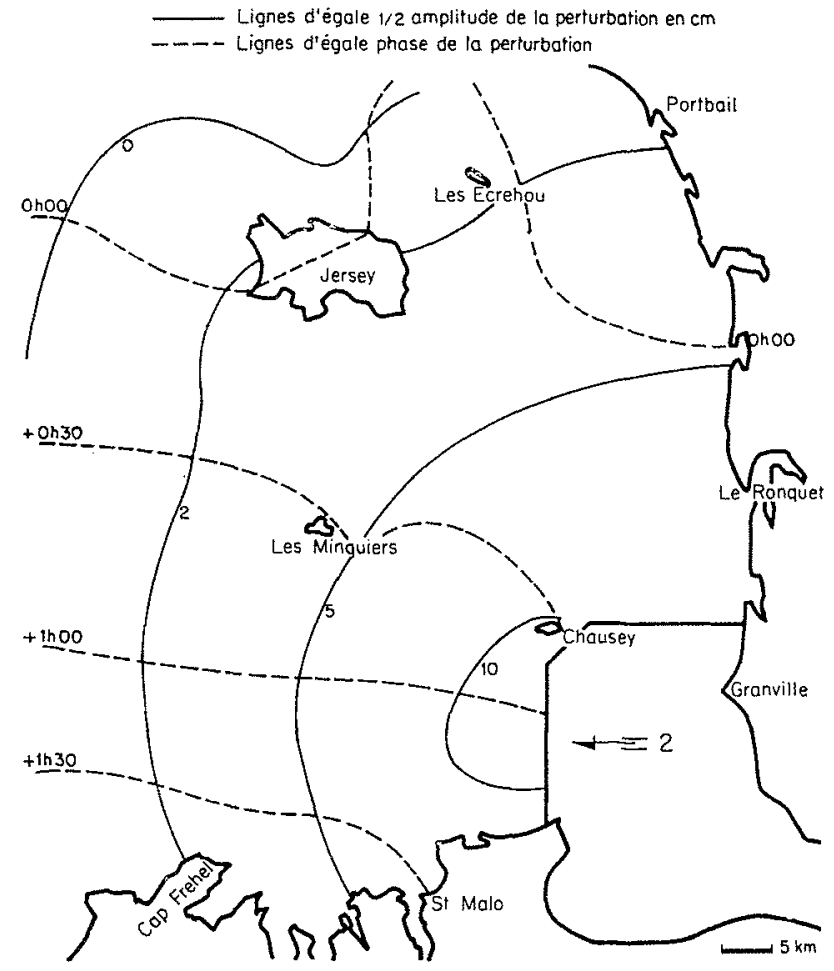

Fig. 9

Influence, sur le niveau moyen de la mer, d'un courant de $1 \mathrm{~m} / \mathrm{s}$ injecté à travers la partie d'usine $\mathrm{n}^{\circ} 2$. Résultats du calcul en négligeant la variation de la pente du fond, mais en tenant compte de la force de Coriolis.

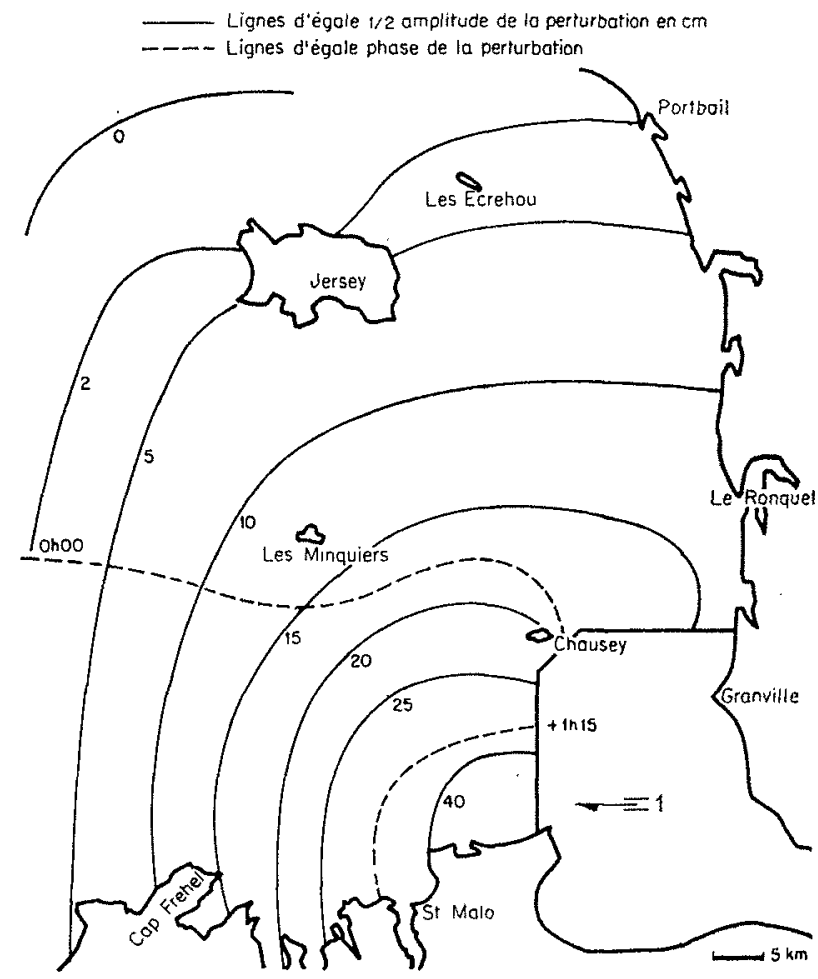

Fig. 11

Influence, sur le niveau moyen de la mer, d'un courant de $1 \mathrm{~m} / \mathrm{s}$ injecté à travers la partie d'usine $n^{\circ} 1$. Résultats du calcul en négligeant la variation de la pente du fond et la force de Coriolis. 


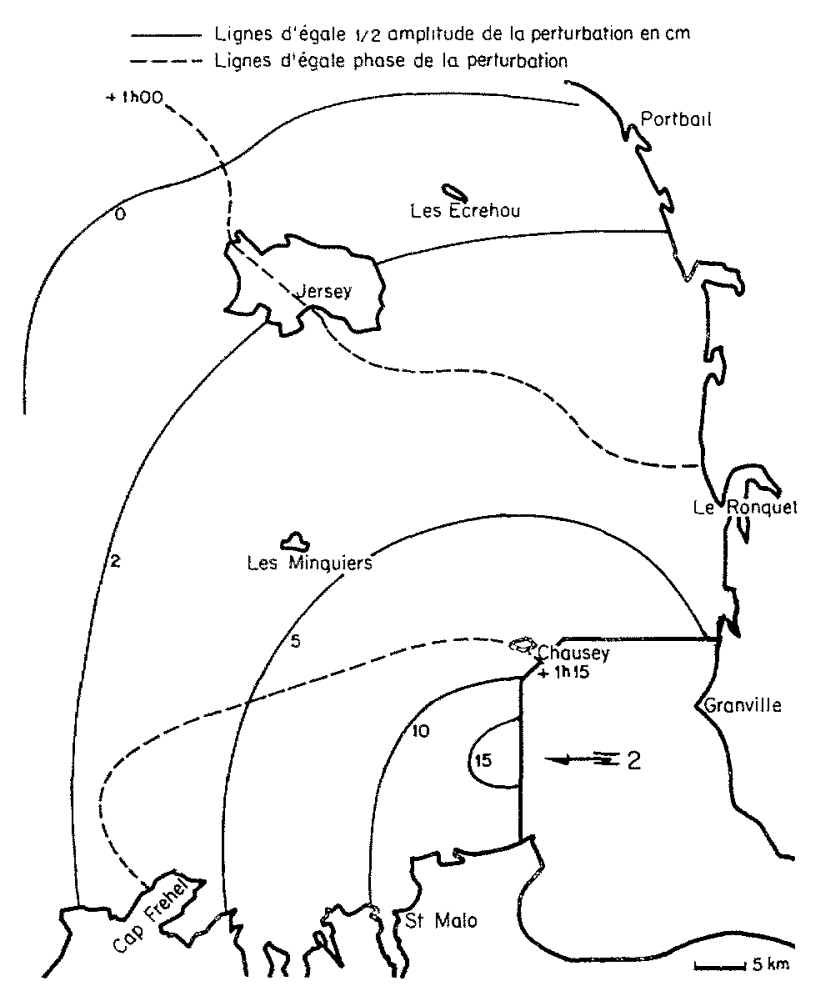

FiG. 12

Influence, sur le niveau moyen de la mer, d'un courant de $1 \mathrm{~m} / \mathrm{s}$ injecté à travers la partie d'usine $\mathrm{n}^{\circ} 2$. Résultats du calcul en négligeant la variation de la pente du fond et la force de Coriolis.

en l'absence de la force de Coriolis, l'onde devrait se propager avec une célérité de l'ordre de $\sqrt{g h}$ (soit $50 \mathrm{~km} / \mathrm{h}$ pour $h=20 \mathrm{~m}$ ), mais le calcul conduit à une propagation plus rapide. D'autre part, on sait que dans un domaine semblable à celui étudié, l'onde soumise à l'effet de la force de Coriolis se déplace du sud au nord et avec une vitesse d'autant plus faible que le frottement sur le fond est lui-même plus faible. Or, pour le calcul, on a introduit un coefficient de frottement linéaire correspondant au courant de marée naturel; il se révèle donc trop faible pour la perturbation étudiée seule, ce qui explique la lenteur de sa propagation due à la proximité d'un point amphidromique.

Les couples de figures 3-6 et 9-12, 4-7 et 10-13, montrent que pour les parties d'usine $\mathrm{n}^{0 \mathrm{~s}} 2$ à 7 ,

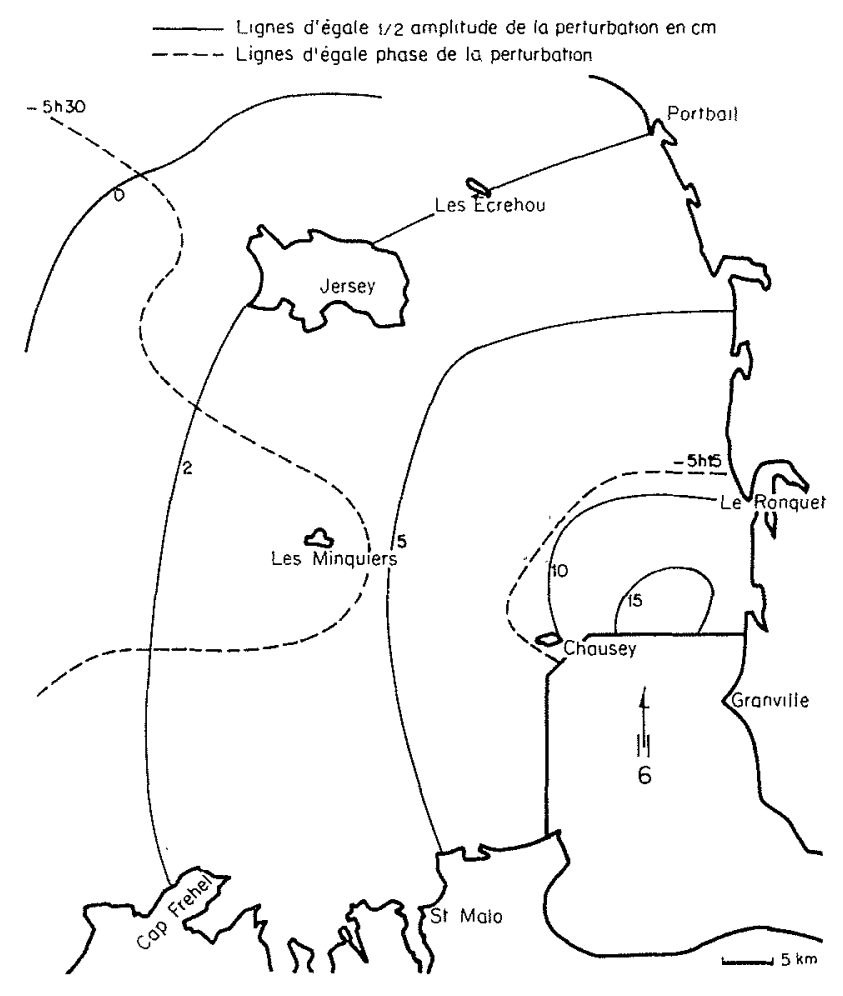

FIG. 13

Influence, sur le niveau moyen de la mer, d'un courant de $1 \mathrm{~m} / \mathrm{s}$ injecté à travers la partie d'usine $n^{\circ} 6$. Résultats du calcul en négligeant la variation de la pente du fond et la force de Coriolis.

la prise en compte de la variation de la pente du fond n'a pas une influence considérable. Par contre, dans le cas de la partie d'usine $n^{\circ} 1$ (fig. 2, 5, 8 et 11), la différence sur l'amplitude de la perturbation est très grande et nous verrons plus loin que les résultats obtenus sur modèle réduit ne confirment pas ce dernier point, alors qu'ils sont en accord avec les autres résultats. Nous pensons que cette divergence provient, d'une part, de ce que le point d'injection $\mathrm{n}^{\circ} 1$ est placé dans une zone où la profondeur varie assez vite, ce qui peut expliquer les grandes valeurs notées sur les figures 8 et 11 et, d'autre part, de la proximité des limites du canevas de calcul dont le maillage local n'est pas parfaitement adapté au calcul. Il faut noter qu'il n'en est pas de même du point d'injection $\mathrm{n}^{0} 7$, pour lequel il n'est pas apparu d'anomalies. 


\section{V. - LE MODELE REDUIT}

En 1954, dès que fut prise la décision d'entreprendre une étude plus poussée de l'usine marémotrice des îles Chausey, un modèle réduit représentant la Manche entière a été construit au Laboratoire National d'Hydraulique. Ce modèle aux échelles de similitude de $1 / 50000$ en plan et 1/500 en hauteur, représente toute la Manche depuis le rebord du plateau continental dans l'Atlantique jusqu'au Pas-de-Calais. La période de la marée est de $20 \mathrm{~s}$ et cette oscillation est engendrée par deux batteurs du type piston disposés aux deux extrémités du modèle (fig. 1). Par suite de sa grande distorsion (100) le modèle a nécessité la mise en place d'un réseau très serré de rugosité pour les besoins de son étalonnage. Mais ce modèle étant fixe, c'est-à-dire lié à la terre, l'accélération complémentaire qui y est induite naturellement par suite de la rotation de la terre est bien trop faible vis-à-vis de celle représentant à l'échelle la même accélération s'exerçant sur la marée naturelle. De ce fait, la propagation de la marée obtenue sur ce modèle diffère sensiblement de celle de la nature, surtout en ce qui concerne les manifestations imputables à la force de Coriolis comme l'existence d'un point amphidromique virtuel et sa consé- quence sur la différence de marnage observée entre les côtes anglaises et françaises.

Cependant l'onde-marée obtenue sur ce modèle est assez correcte dans la région du golfe de Saint-Malo, c'est-à-dire dans la zone intéressée par l'usine marémotrice des îles Chausey. Cette heureuse alternative provient de ce que d'une part, dans l'angle formé par les côtes bretonnes et la presqu'île du Cotentin, les profondeurs sont faibles, donc le frottement est important et que, d'autre part, la barrière du Cotentin constitue une paroi réfléchissante pour l'ondemarée ce qui donne à l'onde-marée un certain caractère stationnaire sur lequel l'effet de la force de Coriolis est moins net. De plus, l'existence de nombreuses iles et goulets étroits confère au régime d'écoulement une grande dépendance de la topographie du bassin évidemment bien représentée sur le modèle réduit. Dans ces conditions, ce modèle réduit a servi de base à l'étude préliminaire de la perturbation de la marée due à la présence et au fonctionnement de l'usine marémotrice des îles Chausey. Nous exposerons ici les résultats de quelques essais effectués dans les conditions les plus analogues à celles correspondant aux calculs exposés cidessus.

\section{VI. - CONDUITE DES ESSAIS}

Sur ce modèle, les oscillations des plans d'eau sont enregistrées, en tout point quelconque, à l'aide de limnigraphes à pointe vibrante asservie avec une précision de l'ordre de $0,1 \mathrm{~mm}$ modèle, ce qui permet de connaître le marnage de la marée reproduite sur le modèle à $5 \mathrm{~cm}$ près.

L'usine est matérialisée sur le modèle par une paroi comportant plusieurs orifices au travers desquels un débit alternatif peut être injecté au point d'injection utilisé pour le calcul numérique. Mais, pour un courant de $1 \mathrm{~m} / \mathrm{s}$, la perturbation obtenue sur le modèle est bien trop faible pour être mesurée avec précision. Dans ces conditions, nous avons d'abord vérifié que cette perturbation est une fonction linéaire de l'am- plitude du courant d'injection dans une gamme assez étendue. Ensuite les mesures de la perturbation ont été effectuées en injectant des cour rants sinusol̈daux correspondant à 3 ou $5 \mathrm{~m} / \mathrm{s}$ selon les points. La perturbation étant alors mesurable, nous avons ramené linéairement sa valeur à celle correspondant à un courant injecté de $1 \mathrm{~m} / \mathrm{s}$. Dans le domaine correspondant à celui des calculs mécaniques, les enregistrements ont été effectués en une trentaine de points; les essais ont été répétés quatre fois et la valeur moyenne a été retenue après avoir constaté une bonne fidélité dans les mesures.

Pour déterminer l'horaire de propagation de 
la perturbation, un top de synchronisation est inserit sur la bande d'enregistrement. Ce top étant émis par le piston fourmissant le débit alternatif, il est possible de connaître la phase du maximum de la perturbation en un point donné par rapport à l'heure du maximum de courant injecté dans le sens bassin-mer. La précision de mesure de cette phase est de l'ordre de $10 \mathrm{mn}$ nature. Pour nos essais, nous avons admis qu'elle était indépendante de l'amplitude du courant injecté, puisque la mesure est effectuée pour un courant de plus de $1 \mathrm{~m} / \mathrm{s}$.

\section{VII. - RESULTATS DES ESSAIS SUR MODËLE REDUIT}

Les cartes des figures 14 à 16 représentent les réseaux de lignes d'égale demi-amplitude et d'égale phase de l'oscillation. Les valeurs moyennes mesurées sont portées à gauche du point de mesure sous forme de la demi-amplitude de l'oscillation en $\mathrm{cm}$, et le déphasage entre cette perturbation et le débit injecté. Le signe + signifie que le maximum de la perturbation a lieu avant le maximum de courant injecté dans le sens bassin-mer.

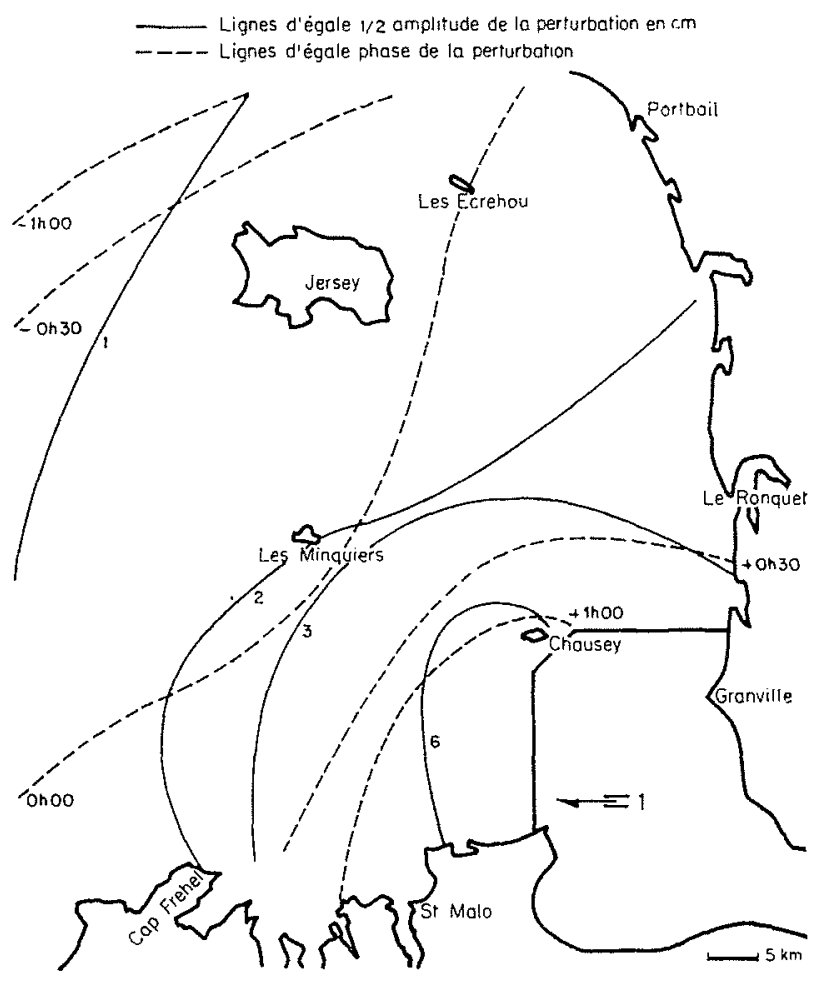

FIG. 14

Influence, sur le niveau moyen de la mer, d'un courant de $1 \mathrm{~m} / \mathrm{s}$ injecté à travers la partie d'usine $\mathrm{n}^{0} 1$. Résultats du modèle réduit.
Rappelons que les essais sur ce modèle réduit correspondent au cas des profondeurs variables, la force de Coriolis étant négligée, et la perturbation n'étant pas forcément nulle le long des limites du canevas de calcul puisque le modèle peut être considéré comme infini. On constate d'ailleurs que la condition d'avoir une amplitude nulle aux limites est pratiquement vérifiée, puisque celle-ci est en général inférieure à $10 \%$ de l'amplitude maximale de l'oscillation.

La figure 14 est théoriquement comparable à la figure 5; on voit qu'en modèle réduit, la propagation de la perturbation est plus lente que pour le calcul; il semble que le frottement étant très fort sur le modèle et très faible pour le calcul, on puisse ainsi expliquer cette différence. En ce qui concerne l'écart sur l'amplitude de la perturbation, il convient de se rappeler que le canevas de calcul au voisinage du point d'injection $\mathrm{n}^{\circ} 1$ ne semble pas convenir. Les figures 15 et 6,16 et 7 respectivement font apparâtre un assez bon accord sur l'allure générale du phénomène. La seule différence provient de la vitesse de propagation de l'onde, mais notons que le modèle réduit conduit bien à une perturbation se propageant à environ $50 \mathrm{~km} / \mathrm{h}$ pour une profondeur de $20 \mathrm{~m}$. Les figures 14 à 16 montrent que, pour le modèle réduit, le maximum de perturbation a toujours lieu au voisinage du point d'injection à $1 \mathrm{~h}$ environ avant le maximum du débit injecté dans le sens bassinmer, ce qui, physiquement, s'explique très bien. Par contre, le calcul conduit à un résultat très différent dans le cas où les points d'injection sont situés sur la face nord de l'usine (fig. 7). Ceci se conçoit mal dans le cas où la force de Coriolis est négligée et où donc il n'y a pas de cause susceptible d'entraîner de dissymétrie du phénomène par rapport à la ligne joignant Jersey au fond de la baie du Mont-Saint-Michel; on notera d'ailleurs que cette dissymétrie ne se manifeste pas sur les marnages. 


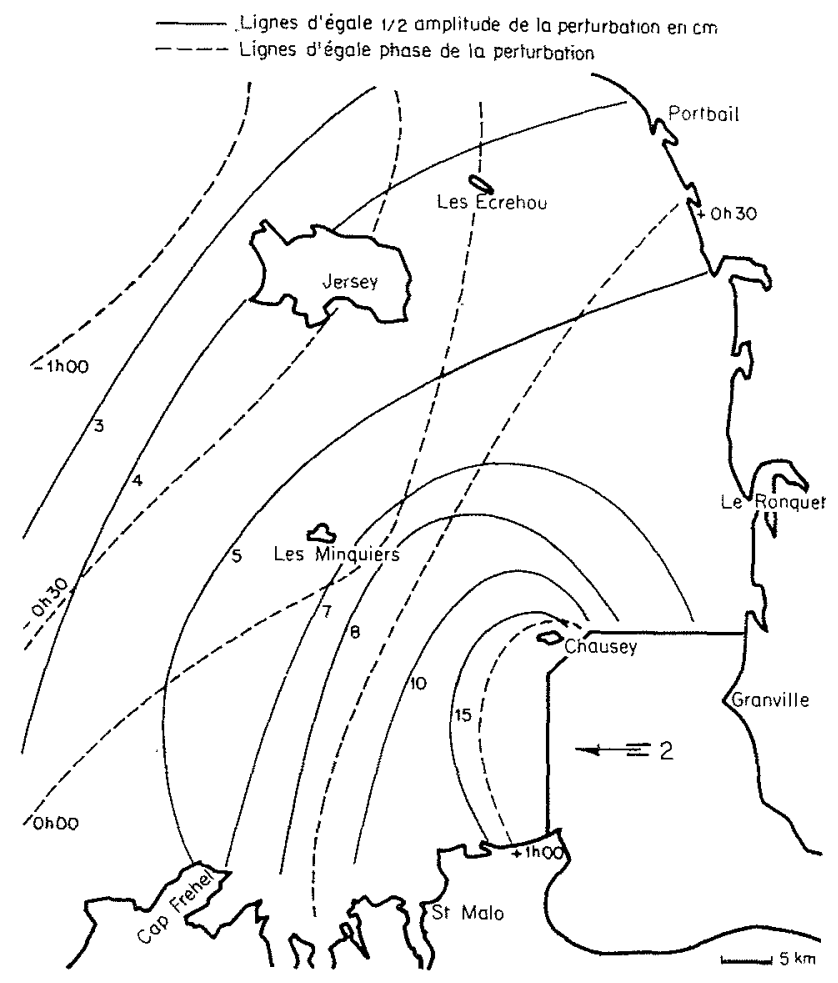

Fig. 15

Influence, sur le niveau moyen de la mer, d'un courant de $1 \mathrm{~m} / \mathrm{s}$ injecté à travers la partie d'usine $\mathrm{n}^{\circ} 2$. Résultats du modèle réduit.

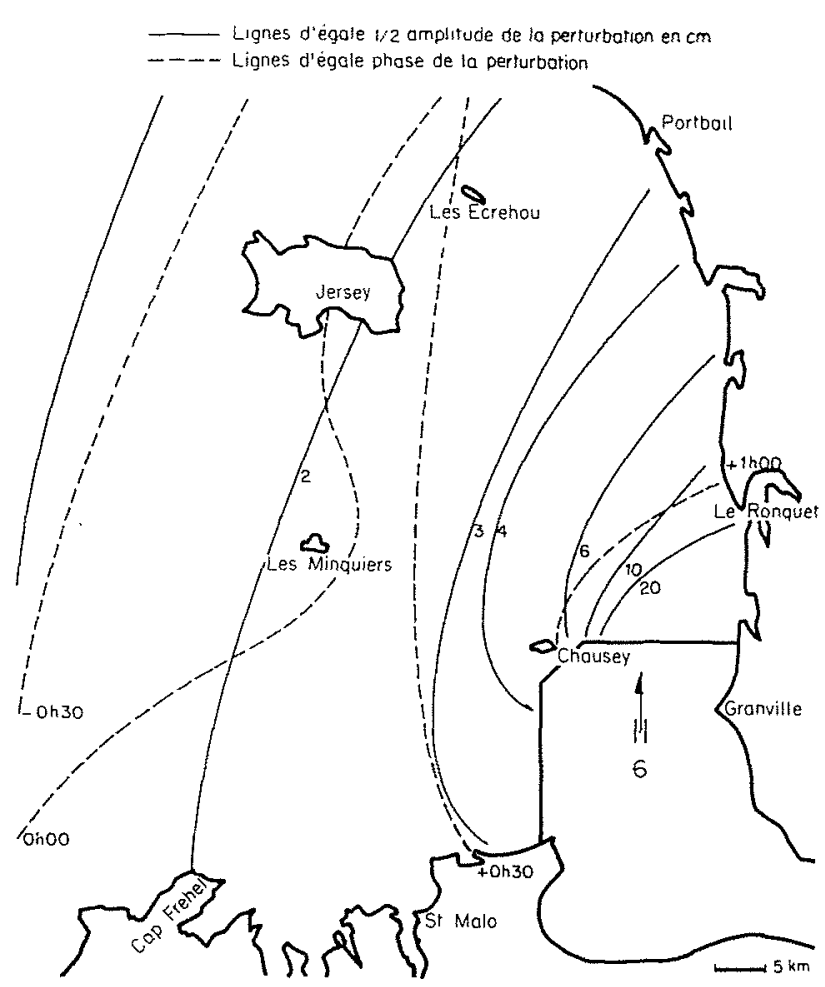

FIG. 16

Influence, sur le niveau moyen de la mer, d'un courant de $1 \mathrm{~m} / \mathrm{s}$ injecté à travers la partie d'usine $n^{\circ} 6$. Résultats du modèle réduit.

\section{VIII. - CONCLUSION}

Les études préliminaires de l'usine marémotrice des îles Chausey ont posé comme première question la détermination de l'influence, sur la marée du golfe de Saint-Malo, de la présence et $\mathrm{du}$ fonctionnement de cette usine. La question a été abordée parallèlement par le calcul et par le modèle réduit. D'une part le calcul revient à effectuer la résolution d'un système de $2 \mathrm{~N}$ équations linéaires représentant la traduction en différences finies de l'équation aux dérivées partielles de la marée en $\mathrm{N}$ points. Nous avons présenté ici les résultats pour $\mathrm{N}=216$ dans plusieurs hypothèses selon que l'on néglige ou non la force de Coriolis ou la pente du fond de la mer. D'autre part, le modèle réduit servant à étudier la perturbation de la marée par l'usine est un modèle représentant toute la Manche; son étalonnage en a été très difficile sinon théoriquement impossible $d u$ fait de ce qu'il ne reproduit pas la force de Coriolis à l'échelle et de sa grande distorsion (100).
La confrontation des résultats du calcul dans différentes hypothèses et de ceux du modèle réduit, sont par certains points décevants, surtout en ce qui concerne la propagation de l'onde représentant la perturbation de la marée due à l'usine, De ce fait, comme les résultats du modèle et ceux du calcul, ne prenant pas en compte la force de Coriolis, ne correspondent pas du point de vue phase, il est difficile d'admettre que la méthode de calcul est parfaitement convenable et qu'elle donne des résultats transposables à la réalité dans le cas où la force de Coriolis est prise en compte comme dans la nature.

Mais, d'un autre côté, la confrontation des résultats modèle et du calcul est très encourageante en ce qui concerne l'amplitude de la perturbation de la marée. Il en résulte que de ce point de vue, les résultats du calcul prenant en compte l'accélération de Coriolis, peuvent être considérés comme valables. Ceci est d'autant 
plus vrai que la perturbation n'a que quelques décimètres d'amplitude, qu'elle est faible visà-vis du marnage local, de lordre de la dizaine de mètres, et qu'il ne suffit pratiquement que de connaître son ordre de grandeur. Considérés sous cet aspect, les résultats des calculs numériques sont alors très utiles et ceci montre combien peut être fructueux le mode de travail en parallèle sur modèles réduits et sur machines à calculer.

C O M M E N T A I R E

de M. le Président BaNaL

M. le Président remercie M. Vorer de son exposé et, tout particulièrement, d'y avoir rappelé si opportunément la part essentielle prise par son chef, notre regrette collègue Lucien Vantrors, à toutes ces études. M. le Président comprend l'émotion de M. Voxer en exposant ces études qui ont toujours été animées par M. VANTroxs, dont il fut l'un des collaborateurs directs.

M. Ie Président présente ensuite les remarques suivantes sur la communication de MM. BonNefille et VOYER :

- Bien que paraissant très sommaire à côté des procédés quasi parfaits qui feront l'objet des communications suivantes, l'étude qui vient d'être présentée a, d'abord, un intérêt historique : elle montre comment l'on a abordé le calcul de la propagation de la marée et rappelle les renseignements et les apaisements que ces premiers résultats ont apportés et qui, finalement, se révèlent n'être pas si loin de la vérité;

- La méthode peut être beaucoup plus perfectionnée, en particulier par une prise en compte plus serrée des fonds et de la côte;

- Mais il reste que cette méthode ne demande que des calculs relativement simples et on peut penser qu'il sera opportun, lorsque des méthodes parfaites auront permis de résoudre d'une manière certaine les problèmes, de reprendre ce calcul pour voir $\mathrm{si}_{\text {, finale- }}$ ment, les difficultés d'ordre théorique qui s'y posent encore, en particulier sur les conditions aux limites, ne peuvent pas être résolues de manière exacte et si, dans beaucoup de cas, on ne peut pas éviter les méthodes trop élaborées et revenir simplement à des calculs de ce genre-là.

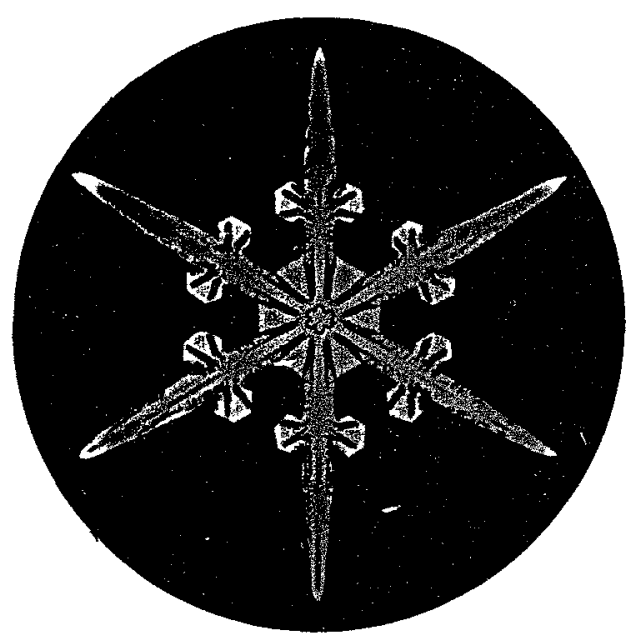

